\title{
Determination of Polyolefine Based Composites Nanostructure Parameters by Very Cold Neutrons Scattering
}

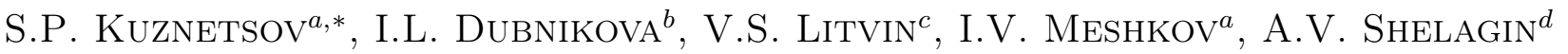 \\ AND A.I. UDOVENKO ${ }^{a}$ \\ ${ }^{a}$ Lebedev Physical Institute, Russian Academy of Sciences, Leninskii Pr. 53, Moscow, 119991 Russia \\ ${ }^{b}$ Semenov Institute of Chemical Physics, Russian Academy of Sciences, Kosygina 4, Moscow, 117977 Russia
}

${ }^{c}$ Institute of Nuclear Researches, Russian Academy of Sciences, Pr. 60 years of October 7A, Moscow, 117312 Russia

${ }^{d}$ Moscow Institute of Physics and Technology, Institutskii per. 9, Dolgoprudny, 141700 Russia

Very cold neutrons (VCN) scattering method was used to study the morphology of polyolefine based nanocomposites. The nanocomposites were prepared by the melt mixing method. The special surface active additions and the fillers surface modification were used to improve the polymer-filler compatibility. The multiwalled carbon nanotubes (MWCNT), the calcium carbonate (CC) submicron particles and the layered natural mineral montmorillonite (MMT) nanoparticles were used as the fillers. The fractal structural model was used to describe the VCN scattering on the nanofillers agglomerates. It was shown by VCN scattering that the volume part of isolated MWCNTs in polypropylene (PP) based composites decreases with the increase of the filler loading, the density of MWCNT packing in agglomerates increases with the total volume nanotube concentration and decreases with the nanotube surface modification. It was revealed that the isolated CC particles are dominated in the $\mathrm{CC} / \mathrm{PP}$ samples with low $\mathrm{CC}$ (5 vol. \%) loading. It was shown that practically full MMT exfoliation within polyethylene (PE) matrix can be achieved by both polymerization exfoliation method and by melt mixing compounding with using special polar polymer additions.

PACS numbers: 61.05.fm, 61.48.De, 82.35.Np

\section{Introduction}

VCN scattering method was developed in Lebedev Physical institute [1] to study nanostructures (NS) of various nature. Last years this method was used to study the NS of novel materials which are created in Semenov Institute of Chemical Physics of the Russian Academy of Sciences. The VCN scattering was successfully used earlier to develop the polymerization exfoliation technology of creation of the polymer nanocomposites based on PE and layered natural mineral montmorillonite, and to develop the technology of creation of the ethylene-norbornene co-polymer with better optical properties compared to origin $\mathrm{PE}$, and so on [2-6].

The properties of polymer nanocomposite materials strongly depend on the particle size and shape as well as on their dispersion state and distribution uniformity within polymer matrix. As it is known, the major obstacles for realization of nanosized particles technological potential is the tendency to clusterization and agglomeration during mixing. In the present work VCN scattering was used to determine the NS parameters of multifunctional polyolefine based nanocomposites with three

* corresponding author; e-mail: ckuz@sci.lebedev.ru nanofiller types - the multiwalled carbon nanotubes, calcium carbonate submicron particles and layered natural mineral montmorillonite nanoparticles.

Two kinds of MWCNTs with external diameters $d<$ $10 \mathrm{~nm}$ (MWCNT1) and with $60 \mathrm{~nm}>d>40 \mathrm{~nm}$ (MWCNT2) - were used for preparation of the multifunctional material with high modulus of elasticity and high electro-and thermo conductivity. The morphologies of two MWCNT/PP composites are illustrated in Fig. 1. The composites NS are characterized by the presence of both isolated MWCNTs and MWCNTs agglomerates. Carbon nanotubes were chemically modified by grafting aliphatic chains onto the MWCNT surface for improving the compatibility between polymer and nanotubes and decreasing the tendency of the nanoparticles to clustering.

Two types of CC submicron particles $(d \cong 100 \mathrm{~nm}$ and $d \cong 60 \mathrm{~nm}$ ) were used as the fillers with the view of preparation of PP composites with the improved balance of elastic modulus-impact toughness. The typical structure of the composite prepared is demonstrated in Fig. 2, showing the presence of both isolated particles and clusters [7]. The special surfactants like calcium stearate, Triton $X-100$, the mixture of fluorine spirits telomeres (FST) were used to improve the polymer - filler compatibility. 

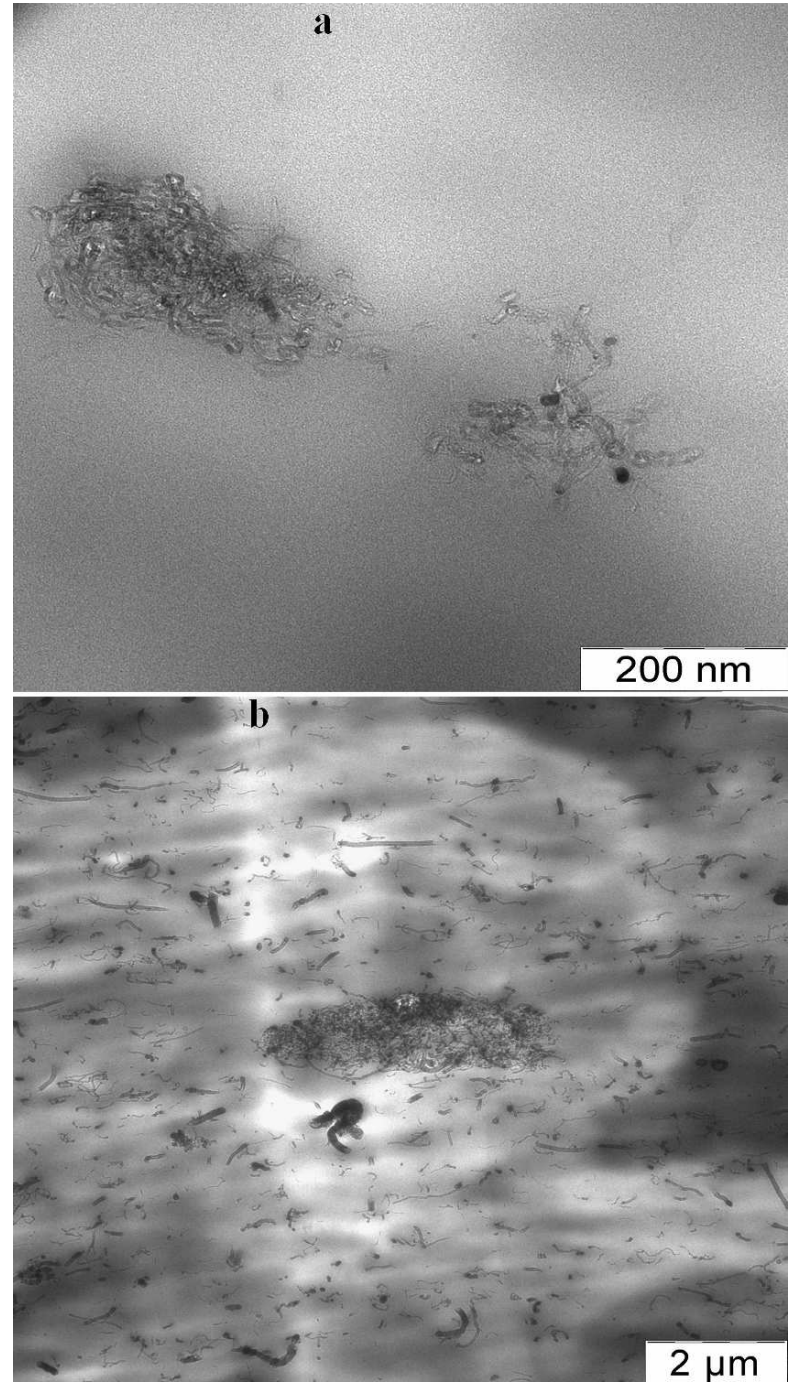

Fig. 1. TEM images of MWCNT1/PP (a) and MWCNT2/PP (b) composites with 1.5 vol. $\%$ filler loading.

The nanocomposites based on high density polyethylene (HDPE) and layered natural mineral montmorillonite were prepared by melt mixing with using maleated HDPE of high (Polybond 3009) and low (Polybond 3109) molecular weight in order to improve the polymer-filler compatibility.

The typical structure of the composite obtained is demonstrated in Fig. 3. The VCN scattering measurements were carried out in order to compare the efficiency of melt mixing and in situ polymerization methods [5] in plane of clay exfoliation.

\section{Method}

Very cold neutrons can be defined as the neutrons with energies below $10^{-2} \mathrm{meV}$ and wavelength values of $\lambda>4.0 \mathrm{~nm}$, respectively. VCN scattering is a convenient tool for NS investigations but the main drawback

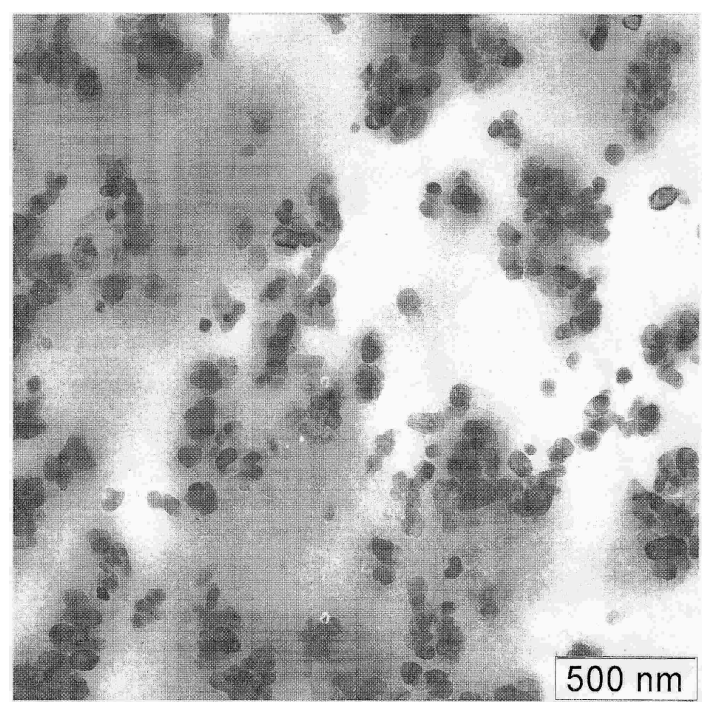

Fig. 2. The typical TEM image of CC/PP composite with 15 vol. \% filler loading.

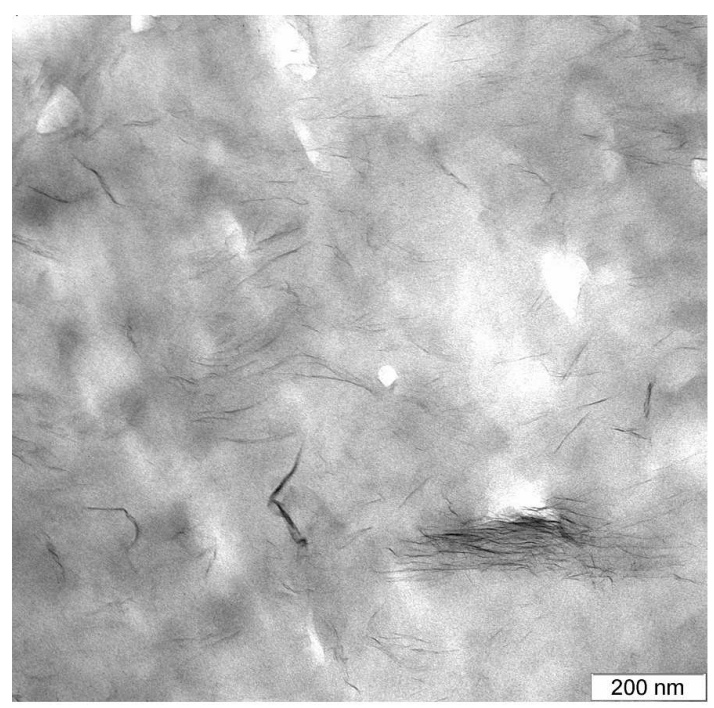

Fig. 3. The typical TEM image of MMT/HDPE composite with 1.5 vol. $\%$ filler loading.

of the VCN application is the low flux on the "tail" of reactor neutrons Maxwell spectrum [1]. Nevertheless, it was shown that using of simple VCN transmission technique give us the possibility to obtain varied information about NS of studied materials [1, 5].

For the sample of thickness $t$ the neutron beam will be attenuated in intensity from $I_{0}$ to $I$ :

$$
I / I_{0}=\exp \left[-t \Sigma_{t}\right], \quad \Sigma_{t}=\Sigma_{\mathrm{a}}+\Sigma_{\mathrm{in}}+\Sigma_{\mathrm{H}}+\Sigma_{\mathrm{NS}},(1)
$$
where $\Sigma_{\mathrm{a}}, \Sigma_{\mathrm{in}}, \Sigma_{\mathrm{H}}, \Sigma_{\mathrm{NS}}$ are the cross-sections for the absorption, inelastic scattering, isotopic and spin-disorder incoherent scattering, and VCN scattering on the NS.

$\Sigma_{\mathrm{NS}}$ can be extracted from $\Sigma_{t}$, if the atomic composition of the studied substance is known and if it is possible to suppress $\Sigma_{\text {in }}$ by cooling. By analogy with small 
angle neutron scattering, using the first Born approximation, differential and integral VCN scattering cross sections can be represented as:

$$
\begin{aligned}
& \mathrm{d} \Sigma_{\mathrm{NS}} / \mathrm{d} \Omega=(A / 2 \pi) \int_{0}^{\infty} K(\rho)[\sin (q \rho) /(q \rho)] \rho^{2} \mathrm{~d} \rho, \\
& \Sigma_{\mathrm{NS}}=\left(2 \pi / k^{-2}\right) \int_{2 k \sin \theta}^{2 k}\left(\mathrm{~d} \Sigma_{\mathrm{NS}} / \mathrm{d} \Omega\right) q \mathrm{~d} q,
\end{aligned}
$$

where $\mathrm{K}(\rho)$ is the correlation function of nuclear-optical potential $U, \Omega$ is solid angle, $A=2 \mathrm{~m}^{2} \hbar^{-4}, m-$ neutron mass, $q$ - modulus of neutron pulse $\boldsymbol{k}$ transfer $q=\left|\boldsymbol{k}-\boldsymbol{k}^{/}\right|, k=|\boldsymbol{k}|, \rho=|\boldsymbol{r}-\boldsymbol{r} /|, \boldsymbol{r}$ - radius vector, $\theta$ - effective angular size of neutron counter.

$K(\rho)$ depends on the parameters of NS (volume part of NS elements, average dimensions of NS elements, etc.) and we can obtain this parameters by comparison of theoretical and experimental dependencies $\Sigma_{\mathrm{NS}}(k)$.

If the characteristic correlation function is used, the analytical expressions for wave vector - or wave lengths - dependences with characteristic parameters of NS can be obtained. The most simple and useful theoretical dependence is $\Sigma_{\mathrm{NS}}(k)$ dependence for isolated spheres with radius $R$ :

$$
\begin{aligned}
\Sigma_{\mathrm{NS}} & =\frac{9 \pi \varphi}{2} T^{2}\left[\frac{4 \pi R^{3}}{3}\right] \frac{1}{k^{2} R^{2}}\left\{\frac{1}{(2 k R \sin \theta)^{2}}\right. \\
& -\frac{\sin (4 k R \sin \theta)}{(2 k R \sin \theta)^{3}}+\frac{\sin ^{2}(2 k R \sin \theta)}{(2 k R \sin \theta)^{4}}-\frac{1}{(2 k R)^{2}} \\
& \left.+\frac{\sin 4 k R}{(2 k R)^{3}}-\frac{\sin ^{2} 2 k R}{(2 k R)^{4}}\right\},
\end{aligned}
$$

$T$ is the $N_{i} b_{i}$ difference on the filler/matrix boundary $\left(\Delta \sum_{i} N_{i} b_{i}\right), \varphi$ - volume part of NS elements in the sample, $N_{i}$ - i-nuclei density, $b_{i}$ - amplitude of the coherent neutron scattering on the i-th nucleus.

In this work we also used the hollow cylinder and thin disc correlation functions [8] in order to describe the VCN scattering on multiwalled carbon nanotubes and nanoparticles of layered natural mineral - montmorillonite in polypropylene or polyethylene matrix. The fractal type correlation function $\xi$ was used to describe VCN scattering on the nanofillers agglomerates in the polyolefine matrix. This correlation function was used earlier $[9]$ to describe a medium where fractals are pores whose sizes are distributed in the range $(R, \xi)$.

$$
K(\rho)=\left(T^{2} D / 4 \pi\right)\left[(R / \rho)^{3-D} \exp (-\rho / \xi)\right]
$$

$D$ - mass-fractal dimension, $\xi$ is increased from 1 to 3 with increasing the density of fillers particles packing in agglomerates.

Thus, $R, \varphi, D$ can be determined by comparison of theoretical and experimental dependencies $\Sigma_{\mathrm{NS}}(k)$. It was difficult to determine the cylinder length $L$ and the fractal maximum size $\xi$ by VCN scattering because $\lambda \ll$ $L, \lambda \ll \xi$ and $\Sigma_{\mathrm{NS}}(k)$ dependency is insensitive to these parameters.

\section{Instruments}

The time-of-flight VCN spectrometer (Fig. 4) was developed [1] in neutron physics department of Lebedev Physical Institute and self in the Research reactor of Moscow Engineering Physics Institute (MEPhI). We use this device to measure $\lambda$ ependences of the VCN total cross sections $\Sigma_{t}(k)$. The neutron vacuum guide tube is used to excrete the VCN from the thermal neutrons reactor spectrum. The mechanical chopper is used to form the neutrons pulses with $1 \mathrm{~Hz}$ frequency in order to provide the time of flight spectrometry. The sample holders connected with cryostat and VCN detector are set in samples chamber. Cryostat is used to decrease the inelastic scattering and to measure at low temperatures.

X-ray Powder diffractometer STADI MP $(\lambda=$ $1.54056 \AA$ ) was used to measure diffraction peaks corresponding to the distance between layers of MWCNT. According to the Debye - Scherrer equation the MWCNT thickness was obtained from the width of diffraction peak corresponding to the distance between MWCNT layers on the half of its maximum (FWHM). We also control the MMT structure exfoliation by X-ray diffraction. The disappearance of diffraction peak corresponding to the distance between MMT layers demonstrates of the full MMT structure exfoliation.

All materials were prepared by melt mixing in a two-roller mixing chamber (Brabender mixer) at $190^{\circ} \mathrm{C}(\mathrm{PP})$ and $170^{\circ} \mathrm{C}$ (HDPE), a rotor rotation speed of $60 \mathrm{rpm}$ and compounding time $15 \mathrm{~min}$. The samples for VCN experiments were prepared by hot pressing.

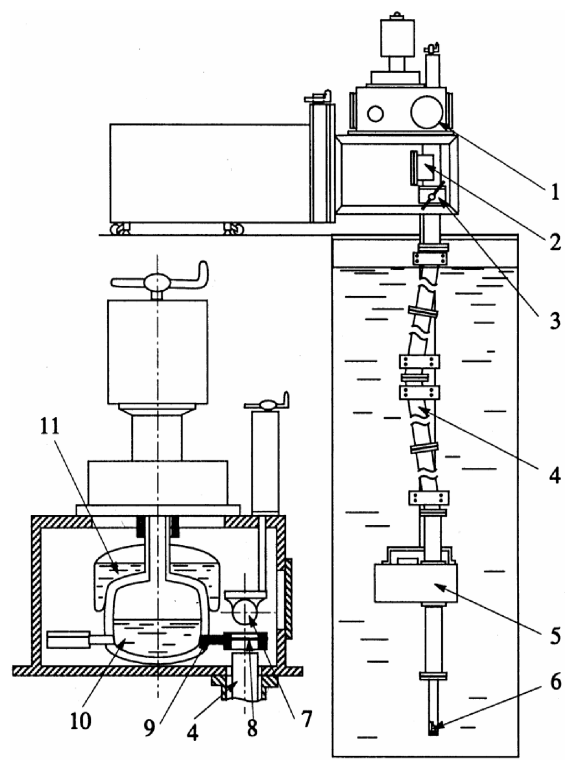

Fig. 4. Very cold neutrons spectrometer. Basic elements: 1 - samples chamber; 2 - neutron counter - monitor; 3 - cut-off valve; 4 - neutron guide tube; 5 - chopper; 6 - converter; 7 - counter; 8 - sample; 9 - sample base; 10 - low-temperature base; 11 nitrogen screen. 


\section{Results}

\section{1. $M W C N T / P P$ composite}

Combination of isolated hollow cylinder [8] and fractal type correlation functions (Eq. 4) was used to describe the VCN scattering on MWCNT/PP samples. The external $(R 1)$ and internal $(R 2)$ radiuses as well as $\varphi$ and $D$ were determined by comparison theoretical and ex- perimental dependencies $\Sigma_{\mathrm{NS}}(k)$. The basic results are represented in the Table I.

Structure parameters of MWCNT/PP composites obtained by VCN scattering method. MWCNTs types: MWCNT1 $-d<10 \mathrm{~nm}$, MWCNT2 - $60 \mathrm{~nm}>d>$ $40 \mathrm{~nm}, \mathrm{~m}-\mathrm{MWCNT}$ - MWCNTs with modified surface. $\chi$ — total volume filler loading, $\varphi$ — volume concentration of isolated MWCNTs.

TABLE I

Structure parameters of MWCNT/PP composites obtained by VCN scattering method. MWCNTs types: MWCNT1 $-d<10 \mathrm{~nm}$, MWCNT2 $60 \mathrm{~nm}>d>40 \mathrm{~nm}$, m-MWCNT MWCNTs with modified surface. $\chi$ - total volume filler loading, $\varphi$ - volume concentration of isolated MWCNTs.

\begin{tabular}{c|c|c|c|c}
\hline \hline $\begin{array}{c}\text { MWCNT } \\
\text { type }\end{array}$ & $\begin{array}{c}\chi \\
\text { (producer) }\end{array}$ & $\begin{array}{c}R 1(R 2),[\mathrm{nm}] \\
(\mathrm{VCN} \text { method) }\end{array}$ & $\begin{array}{c}\varphi / \chi, \% \\
(\text { VCN method) }\end{array}$ & $\begin{array}{c}D \\
\text { (VCN method) }\end{array}$ \\
\hline MWCNT1 & 0.5 & $4.0(1.0)$ & 14 & 2.7 \\
\hline \multirow{2}{*}{ m-MWCNT1 } & 0.5 & $4.0(1.0)$ & 20 & 2.5 \\
\cline { 2 - 5 } & 1.5 & $4.4(1.4)$ & 20 & 2.5 \\
\hline \multirow{2}{*}{ MWCNT2 } & 0.5 & $23(18)$ & 60 & 1.55 \\
\cline { 2 - 5 } & 1.5 & $23(14)$ & 36 & 2.02 \\
\hline \multirow{2}{*}{ m-MWCNT2 } & 0.5 & $23(18)$ & 60 & 1.38 \\
\cline { 2 - 5 } & 1.5 & $25(15)$ & 33 & 2.02
\end{tabular}

TABLE II

Structure parameters of CC/PP composites obtained by VCN scattering method.

Surfactant nature: 1 - calcium stearate; 2 - Triton X-100; 3 - FST.

\begin{tabular}{c|c|c|c|c|c}
\hline \hline $\begin{array}{c}R 1,[\mathrm{~nm}] \\
\text { (producer) }\end{array}$ & $\begin{array}{c}\chi, \text { vol. } \% \\
\text { (producer) }\end{array}$ & $\begin{array}{c}R 1,[\mathrm{~nm}] \\
\text { (VCN method) }\end{array}$ & $\begin{array}{c}\varphi / \chi, \text { vol. } \% \\
\text { (VCN method) }\end{array}$ & $\begin{array}{c}D \\
\text { (VCN method) }\end{array}$ & $\begin{array}{c}\text { Surfactant } \\
\text { nature }\end{array}$ \\
\hline 50 & 5 & 47.0 & 90 & 2.8 & - \\
50 & 15 & 39.0 & 30 & 2.85 & 1 \\
50 & 15 & 48.0 & 26 & 2.85 & 2 \\
50 & 15 & 48.0 & 30 & 2.87 & 3 \\
30 & 5 & 28.0 & 90 & 2.8 & 2
\end{tabular}

TABLE III

The exfoliation degree of MMT in HDPE composites evaluated by VCN scattering method.

\begin{tabular}{c|c|c|c|c|c|c}
\hline \hline Sample & $\begin{array}{c}\chi, \text { vol. \% } \\
\text { (producer) }\end{array}$ & $\begin{array}{c}\varphi, \text { vol. } \% \\
(\mathrm{VCN})\end{array}$ & $\begin{array}{c}\text { Exfoliation } \\
\varphi / \chi, \%\end{array}$ & \multicolumn{2}{|c|}{$\begin{array}{c}\text { Special polar polymer } \\
\text { additions }\end{array}$} & $\begin{array}{c}\text { X-ray diffraction } \\
\text { peak presence }\end{array}$ \\
\cline { 5 - 6 } & & & & type & content, $\%$ & \\
\hline 1 & 0.5 & 0.45 & 90 & - & 0 & - \\
2 & 1.6 & 1.54 & 97 & PB3109 & 100 & no \\
3 & 1.6 & 0.83 & 50 & PB3109 & 8 & yes \\
4 & 1.6 & 1.0 & 65 & PB3009 & 8 & weak \\
5 & 3.8 & 1.52 & 58 & PB3009 & 19 & yes
\end{tabular}

It was shown that the external radiuses determined by $\mathrm{VCN}$ are in the region declared by nanotube producer (Shenzhen Nanotech Port Co. China), the volume part of isolated tubes $(\varphi / \chi)$ decreases with the increase of the total volume loading of carbon nanotubes as well as with the decrease of the nanotube external radius, the density 
of agglomerates packing increases with the total volume nanotubes concentration. Some effects of nanotube surface modification on mass-fractal dimension, especially for samples with low nanotube volume loading, were determined.

The thicknesses of the nanotubes were obtained as the difference between external and internal radiuses and the values calculated are $3 \mathrm{~nm}$ for thin MWCNT1 nanotubes and 5-10 nm for thick MWCNT2 nanotubes. The thicknesses of nanotubes determined by the X-ray diffraction are 3-4 nm for MWCNT1 and 7-8 nm for MWCNT2.

\section{2. $C C / P P$ composite}

In this case the theoretical $\Sigma_{\mathrm{NS}}(k)$ dependences were obtained by combination of isolated sphere (Eq. 3) and fractal type correlation functions (Eq. 4). The average radius and volume part of the isolated CC particles as well as the fractal mass dimension were determined (see Table 2). It was determined that the volume part of the isolated filler particles decreases with the increase of the total filler loading and it is independent on the surfactant type. It is interesting that the mass fractal dimension is also independent on the surfactant type as well as on the total filler loading.

Structure parameters of $\mathrm{CC} / \mathrm{PP}$ composites obtained by VCN scattering method. Surfactant nature: 1 - calcium stearate; 2 - Triton X-100; 3 - FST.

\section{3. $M M T / P E$ composites}

The thin isolated disc correlation function [8] was used in order to describe the VCN scattering on MMT nanoparticles in polyethylene matrix. Volume part of isolated MMT particles was determined by minimization of the discrepancy between the experimental and theoretical dependences $\Sigma_{\mathrm{NS}}(k)$. The typical dependence is presented in Fig. 5.

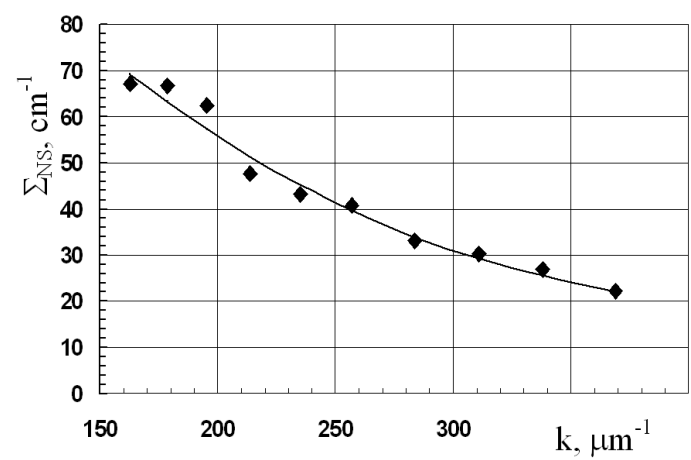

Fig. 5. Theoretical (-) and experimental $(\bullet) \Sigma_{\mathrm{NS}}(k)$ dependences obtained for $\mathrm{MMT} / \mathrm{PE}$ sample No. 2 (Table III).

It was shown (Table III) that the highest exfoliation was achieved at melt mixing for composite based on the maleinated HDPE (PB3109) and for the sample obtained by polymerization exfoliation method (Sample 1). Increasing the MMT exfoliation degree at the same filler loading in composite with PB3009 and decreasing that characteristic with increasing filler loading were shown too.

These experiments were made in order to compare the exfoliation MMT intensity during both the simple melt mixing and in situ polymerization [2] nanocomposites preparation methods. The exfoliation was also controlled by X-ray diffraction. Disappearance of diffraction peak corresponding to the distance between MMT layers was the argument of the high exfoliation degree of the MMT layered structure and was in agreement with VCN date. Thus it was shown that high MMT exfoliation degree within polymer matrix can be achieved both by polymerization intercalation method and by melt mixing in the presence of polar polymer additions.

\section{Conclusions}

VCN scattering method was successfully used in the process of polyolephyne based nanocomposites creation. Fractal structure model was successfully used in investigations of nanoparticles dispersion state and agglomeration in polymer matrix. The important information about distribution of nanofillers in the polymer matrix was obtained. In particular the volume fraction of isolated MWCNTs decreases with the decrease of nanotube diameter. It was shown for all samples that the volume fraction of isolated nano filler particles decreases with the increase of filler loading. It was demonstrated that surface modification of MWCNT leads to the decrease of the agglomerate density at low (0.5 vol. \%) filler loading.

Any noticeable effect of surfactants used on the submicron particle agglomeration in the $\mathrm{CC} / \mathrm{PP}$ composites were not found.

It was shown that using of the polar polymer additions in the process of nanocomposite preparation by melt mixing method leads to the substantial increase of the MMT exfoliation degree in MMT/PE composites.

\section{Acknowledgments}

We would like to express our gratitude to Yu.A. Lapushkin for his participation in VCN-spectrometric measurements, and to colleagues from the Moscow Engineering Physics Institute and Semenov Chemical Physics Institute for assistance and interest in this work. We also wish to thank Dr. Sergey S. Abramchuk from Moscow State University for the great assistance in transmission electron microscopy analysis.

This work was supported by the Program of fundamental researches of the Physical Sciences Department of the Russian Academy of sciences.

\section{References}

[1] A.V. Antonov, A.I. Isakov, S.P. Kuznetsov, I.V. Meshkov, A.D. Perekrestenko, A.V. Shelagin, Fizika Tverdogo Tela 26, 1585 (1984). 
[2] N.Yu. Kovaleva, P.N. Brevnov, V.G. Grinev, S.P. Kuznetsov, I.V. Pozdnyakova, S.N. Chvalun, E.A. Sinevich, L.A. Novokshonova, Polymer Science A 46, 651 (2004).

[3] S. Kuznetsov, Yu. Lapushkin, A. Mitrofanov, S. Shestov, A. Udovenko, A. Shelagin, I. Meshkova, V. Grinev, E. Kiseleva, L. Raspopov, A. Shchegolikhin, L. Novokshonova, Crystallography Reports 52, 496 (2007).

[4] I.N. Meshkova, V.G. Grinev, E.V. Kiseleva, L.N. Paspopov, S.P. Kuznetsov, A.I. Udovenko, A.N. Shchegolikhin, T.A. Ladygina, L.A. Novokshonova, Polymer Science A 49, 1165 (2007).

[5] I.N. Meshkova, V.G. Grinev, L.N. Raspopov, A.N. Shchegolikhin, S.P. Kuznetsov, A.V. Mitrofanov, S.V. Shestov, A.I. Udovenko, L.A. Novokshonova, Polimery LIII, 345 (2008).
[6] S.P. Kuznetsov, A.D. Perekrestenko, Investigations with very cold neutrons on the MEPhI reactor, Ingenernaya Fizika, On the 40th anniversary of MEPhI reactor physical start, 2, 39 (2007).

[7] I.L. Dubnikova, E.I. Nizhegorodseva, S.M. Lomakin, V.G. Krasheninnikov, A.Ya. Gorenberg, V.N. Kuleznev, Polymer Science A 50, 1214 (2008).

[8] G. Porod, Acta Phys. Aust. B 2, 255 (1948).

[9] S.V. Maleev, Phys. Rev. B 52, 13163 (1995). 